\title{
A Decoupling Control Method for Shunt Hybrid Active Power Filter Based on Generalized Inverse System
}

\author{
$\mathrm{Xin} \mathrm{Li}^{1,2}$ and $\mathrm{Bo} \mathrm{Li}^{2}$ \\ ${ }^{1}$ Key Laboratory of Optoelectronic Technology and Intelligent Control, Ministry of Education, Lanzhou Jiaotong University, \\ Lanzhou 730070, China \\ ${ }^{2}$ School of Automation \& Electrical Engineering, Lanzhou Jiaotong University, Lanzhou 730070, China \\ Correspondence should be addressed to Xin Li; lxfp167@163.com
}

Received 13 April 2017; Accepted 15 June 2017; Published 24 July 2017

Academic Editor: Ephraim Suhir

Copyright (C) 2017 Xin Li and Bo Li. This is an open access article distributed under the Creative Commons Attribution License, which permits unrestricted use, distribution, and reproduction in any medium, provided the original work is properly cited.

In this paper, a novel decoupling control method based on generalized inverse system is presented to solve the problem of SHAPF (Shunt Hybrid Active Power Filter) possessing the characteristics of 2-input-2-output nonlinearity and strong coupling. Based on the analysis of operation principle, the mathematical model of SHAPF is firstly built, which is verified to be invertible using interactor algorithm; then the generalized inverse system of SHAPF is obtained to connect in series with the original system so that the composite system is decoupled under the generalized inverse system theory. The PI additional controller is finally designed to control the decoupled 1-order pseudolinear system to make it possible to adjust the performance of the subsystem. The simulation results demonstrated by MATLAB show that the presented generalized inverse system strategy can realise the dynamic decoupling of SHAPF. And the control system has fine dynamic and static performance.

\section{Introduction}

In recent years, many nonlinear electrical components are widely used in our life; harmonics produced by Power System electronic devices have a serious impact on power quality. So it is important to find equipment to suppress harmonics and compensate reactive power for the grid.

The traditional compensation equipment is passive filter (PPF); it is inexpensive, but it cannot solve the impedance deviation caused by the mutation of the system, and it can only compensate fixed harmonics. Active power filter (APF) can be used to compensate each of the order harmonics of the grid, but it is not so feasible in large-capacity situation [1].

As a new compensation device, HAPF combines the advantages of PPF and APF; it can solve almost all the matters that PPF and APF have. Harmonics and reactive power are compensated by PPF. The main function of APF is to improve the filtering characteristics of passive filters and overcome the shortcomings that the PPF has which can easily resonate with the grid impedance. The capacity of APF occupies only about $2 \%-5 \%$ of the capacity of the harmonic source load, and most harmonics are compensated by PPT. So SHAPF can be used in large-capacity situations with relatively small-capacity, and it can improve the cost effectiveness of the system. So the use of SHAPF is becoming a significant practical direction for grid harmonic governance.

The design of the controllers seriously affects the harmonics compensation. Many controllers have been designed to make the APF decoupling. Reference [1] designed a double closed-loop PI controller to achieve the decoupling control. Reference [2] designed a fuzzy controller to achieve the decoupling control, but the settings of controllers mainly depend on experience, and the controllers cannot react to the mutation of the system. In [3], inverse system method is presented to achieve the decoupling of SHAPF. The compensation effect is better, but the traditional analytic inverse system method largely depends on the accurate mathematical model of the system, and it is unable to configure the poles of the system to achieve the expected performance of the system. In this paper, the traditional inverse system method is improved and a new generalized inverse decoupling control system is built. Finally we can ensure the stability of the 


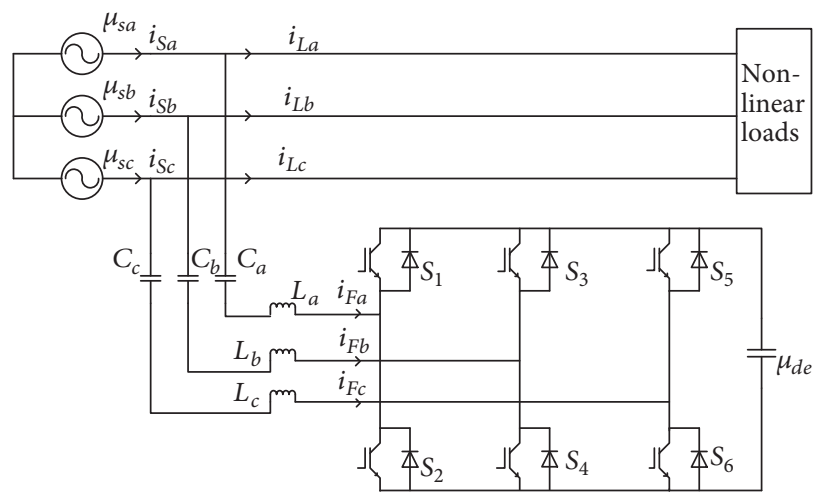

FIgURE 1: Mathematical model of SHAPF [1].

pseudolinear system and reduce the difficulty of additional controller design, by adjusting the generalized inverse parameters to configure the pole of the system.

\section{Working Principle of SHAPF}

SHAPF consists of command current arithmetic circuit, current tracking control circuit, driving circuit, and the main circuit (PWM converter). The structure of SHAPF is shown in Figure 1. By detection of the harmonic current on grid, compensated current signal is firstly calculated by compensated amplifier circuit. Then PWM converter generates compensated current by driving circuit; compensated current can offset the harmonic current on grid. Finally, expected current is obtained by PWM generator. The core device of SHAPF main circuit is IGBT (Insulated Gate Bipolar Transistor), it is widely used because of its high frequency inverter characteristic and automatic switching current capability, and it can transform the direct current (DC) into alternating current (AC). SHAPF is equivalent to the controlled current source in the main circuit; it is used to offset the harmonics and reactive power on the grid [4].

\section{Mathematical and Generalized Inverse of SHAPF}

The mathematical model of SHAPF is shown as Figure 1. $v_{s j}$ $(j=a, b, c)$ is grid voltage. $v_{c j}(j=a, b, c)$ is the voltage of $C_{j}$ $(j=a, b, c) \cdot v_{l j}(j=a, b, c)$ is the voltage of point of common coupling. $L_{s}$ is the equivalent inductance of the grid. $C_{d e}$ is the DC capacitor and the voltage is $v_{d e}$.

From [5], we get the formula of SHAPF in $d$ - $q$ coordinate system:

$$
\begin{aligned}
& L_{F} i_{F d}^{\prime}=-R_{F} i_{F d}+\omega L_{F} i_{F q}-v_{C d}-u_{d} v_{d c}+v_{L d} \\
& L_{F} i_{F q}^{\prime}=-R_{F} i_{F q}-\omega L_{F} i_{F d}-v_{C q}-u_{q} v_{d c}+v_{L q} \\
& C_{d e} v_{d c}^{\prime}=u_{d} i_{F d}+u_{q} i_{F q} .
\end{aligned}
$$

For the facilitation of the controller design, nonlinear affine expression of SHAPF is built as follows:

$$
\begin{aligned}
x^{\prime} & =f(x)+g(x) u \\
y & =h(x) .
\end{aligned}
$$

Providing that $x=\left[\begin{array}{lll}i_{F d} & i_{F q} & v_{d c}\end{array}\right]^{T}, u=\left[\begin{array}{ll}u_{d} & u_{q}\end{array}\right]^{T}$, and $y=\left[\begin{array}{ll}y_{1} & y_{2}\end{array}\right]^{T}=\left[\begin{array}{ll}i_{F d} & i_{F q}\end{array}\right]^{T}$, we can get the derived formula from formula (1)-(2):

$$
\begin{aligned}
& x_{1}^{\prime}=\frac{\left(-R_{F} x_{1}+\omega L_{F} x_{2}-v_{C d}-u_{1} x_{3}+v_{L d}\right)}{L_{F}} \\
& x_{2}^{\prime}=\frac{\left(-R_{F} x_{2}-\omega L_{F} x_{1}-v_{C q}-u_{2} x_{3}+v_{L q}\right)}{L_{F}} \\
& x_{3}^{\prime}=u_{1} x_{1}+u_{2} x_{2} .
\end{aligned}
$$

In formula (1)-(6), $\omega$ is the fundamental angular frequency. In other words, it is the rotation angular velocity of the $d-q$ axis coordinate; $u_{1}$ and $u_{2}$ are the switching function control quantities in $d-q$ axis coordinate; $v_{L d}$ and $v_{L q}$ are the voltage at the connection point between AC and SHAPF in $d-q$ axis coordinate. $i_{d}$ and $i_{q}$ are the compensation current of SHAPF in $d-q$ axis coordinate. We can get the following formula from (9)-(11). From formula (5)-(6), it is easy to see that the system is a 2-input-2-output coupling and nonlinear system. In this paper, the generalized inverse system method is used to design the controller.

\section{Generalized Inverse Controller Design for SHAPF}

4.1. Invertible Analysis. Here, we use inverse system to analyze the invertibility of SHAPF. We first calculate the derivative of the output $(y)$ until the input $(u)$ appears explicitly [6]. In this paper, calculating $y=h(x)^{\prime}$,

$$
\begin{aligned}
& h_{1}(x)^{\prime}=\frac{\left(-R_{F} x_{1}+\omega L_{F} x_{2}-v_{c d}-u_{1} x_{3}+v_{L d}\right)}{L_{F}} \\
& h_{2}(x)^{\prime}=\frac{\left(-R_{F} x_{2}-\omega L_{F} x_{1}-v_{c q}-u_{2} x_{3}+v_{L q}\right)}{L_{F}} .
\end{aligned}
$$

The Jacobi matrix is $\left[\begin{array}{cc}-v_{d e} / L_{F} & 0 \\ 0 & -v_{d e} / L_{F}\end{array}\right]$.

The Jacobi matrix is nonsingularity; relative degree vector of the system is $\alpha=\left[\alpha_{1}, \alpha_{2}\right]^{T}=[1,1]^{T}$. For $\alpha_{1}=\alpha_{2}=1, \alpha_{1}+$ $\alpha_{2}=2$, the order is less than the order of the SHAPF system, so the SHAPF system is invertible. It is confirmed in [7]. 


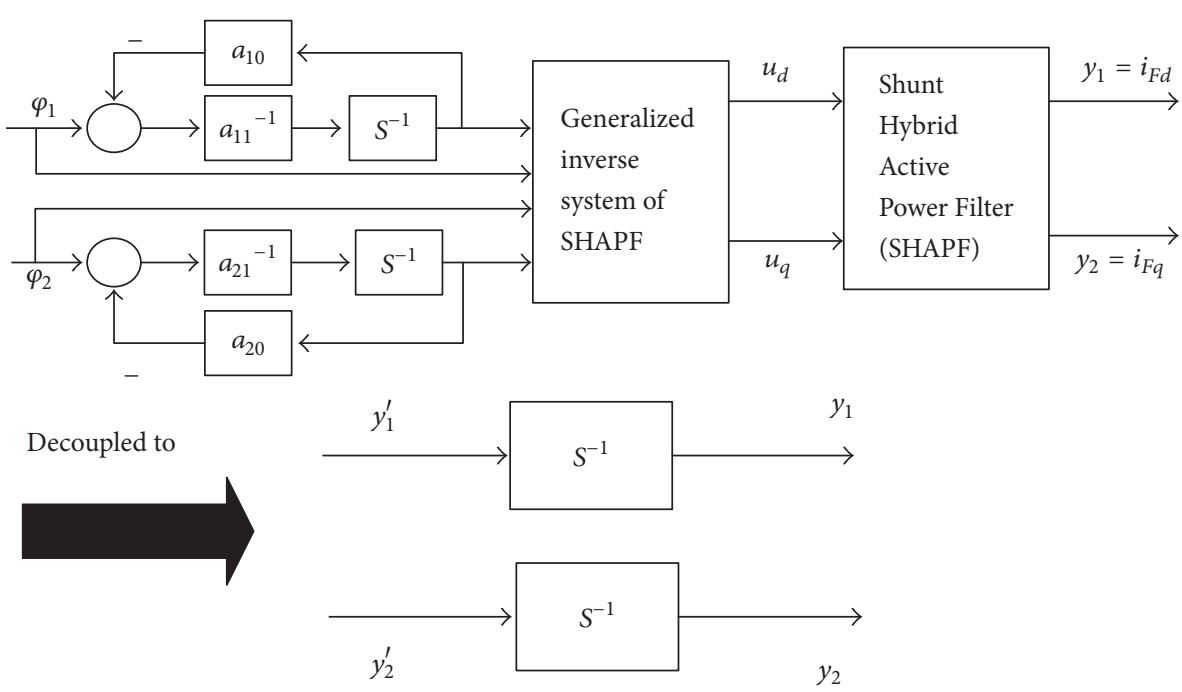

FIGURE 2: Feedback linearization and decoupling of SHAPF with generalized inverse system method.

4.2. Formula Derivation with Generalized Inverse Theory. As is proved in the previous Section 4.1, the system is invertible. In inverse system, the input vector of the inverse system is $\varphi=$ $y^{\prime}$; the output vector of the inverse system is $\left[\begin{array}{ll}y_{1} & y_{2}\end{array}\right]^{T}$ and the state feedback vector of the inverse system is $\left[\begin{array}{ll}x_{1} & x_{2}\end{array}\right]^{T}$. By generalized inverse system, the input vector changes to $\varphi_{j}=$ $a_{j_{0}} y_{0}+a_{j 1} y_{1}^{\prime}+a_{j 2} y_{2}^{\prime \prime}+\ldots+a_{j n} y_{j n}^{(n)}$.

It is obvious that, compared with the inverse system, generalized inverse system increases some parameters, so the pseudolinear system can configure the poles to anywhere by adjusting parameters. It cannot be resolved by inverse system. So the decoupling effect by generalized inverse system is better than the traditional inverse system.

In this paper, ordering the input vector $\varphi$ is

$$
\begin{aligned}
& \varphi_{1}=a_{11} y_{1}^{\prime}+a_{10} y_{1} \\
& \varphi_{2}=a_{21} y_{2}^{\prime}+a_{20} y_{2} .
\end{aligned}
$$

We transform (9)-(10) into

$$
\begin{aligned}
& y_{1}^{\prime}=\frac{\left(\varphi_{1}-a_{10} y_{1}\right)}{a_{11}} \\
& y_{2}^{\prime}=\frac{\left(\varphi_{2}-a_{20} y_{2}\right)}{a_{21}} .
\end{aligned}
$$

From (5)-(6), we can get the expression of $u_{d}$ and $u_{q}$ :

$$
\begin{aligned}
& u_{d}=\frac{\left(v_{L d}-R_{F} x_{1}+\omega L_{F} x_{2}-v_{C d}-L_{F} y_{1}^{\prime}\right)}{x_{3}} \\
& u_{q}=\frac{\left(v_{L q}-R_{F} x_{2}-\omega L_{F} x_{1}-v_{C q}-L_{F} y_{2}^{\prime}\right)}{x_{3}} .
\end{aligned}
$$

Substituting (11)-(12) into equation (13):

$$
\begin{aligned}
& u_{d} \\
& =\frac{\left(v_{L d} a_{11}-R_{F} x_{1} a_{11}+\omega L_{F} x_{2} a_{11}-v_{c d} a_{11}+L_{F} a_{10} x_{1}-L_{F} \varphi_{1}\right)}{\left(a_{11} x_{3}\right)} \\
& u_{q}=\frac{\left(v_{L q} a_{21}-R_{F} x_{2} a_{21}-\omega L_{F} x_{1} a_{21}+L_{F} a_{21} x_{2}-L_{F} \varphi_{2}\right)}{\left(a_{21} x_{3}\right)} .
\end{aligned}
$$

Equation (14) is the duty cycle function of PWM generator for the new generalized inverse system. Feedback linearization and decoupling of SHAPF with generalized inverse system method diagram are shown in Figure 2. We can control the compensated current by adjusting the duty cycle function of PWM inverter. By this way, the control of 2input-2-output system is transformed into the control of two linear systems, so the 1-order linear system is easy to control.

4.3. Design of Controller of Pseudolinear System. With generalized inverse system method, the controller is designed to achieve the desired effect. In this paper, generalized inverse PI composite controller is designed to achieve the decoupling control; two PI additional controllers are used to control the two decoupled 1-order pseudolinear systems which can reduce steady-state error of the system and configure the poles reasonably. The capacitor in DC side is controlled by PI control. Simulation flow chart of generalized inverse system is shown in Figure 3.

4.4. Adjustment of Generalized Inverse System Parameters. The composite system is a second-order system, structure diagram of SHAPF control system is shown in Figure 4. The transfer function of the system is easy to obtain. It is calculated that the poles of the composite system are all on the left side of the $s$-plane, so the composite system is stable. The generalized inverse system parameters $\left(a_{10}, a_{11}, a_{20}, a_{21}\right)$ 


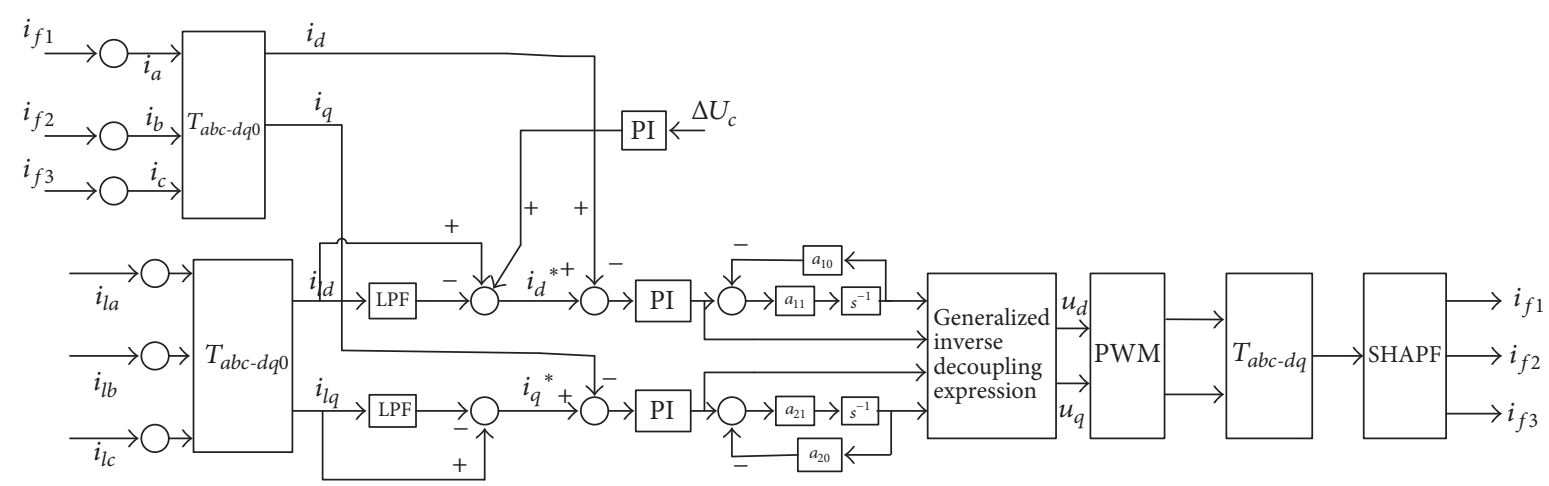

FIGURE 3: Simulation flow chart of generalized inverse system.

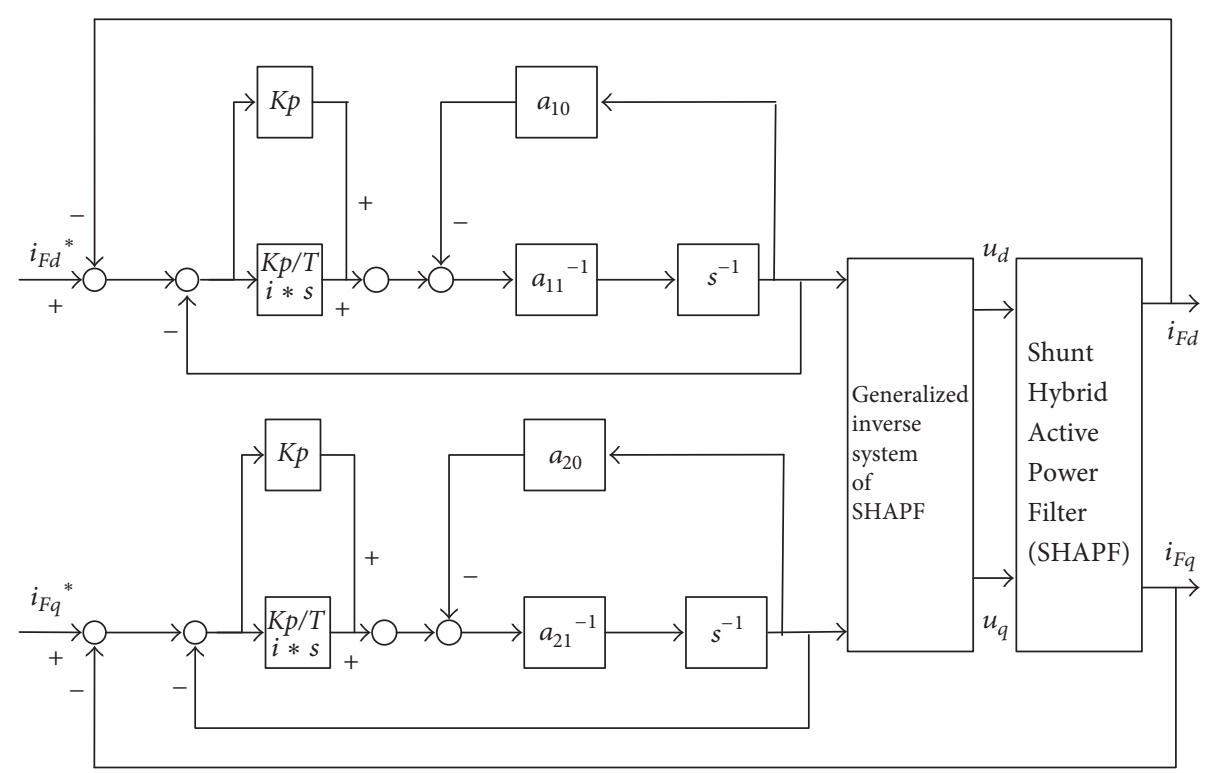

FIGURE 4: Structure diagram of SHAPF control system.

are set to make the damping ratio $(\xi)$ of this composite subsystem close to 0.7 . (The damping ratio is calculated by the transfer function.) The operating frequency range can reach to the maximum value, and the subsystem has the best overall dynamic performance. This best damping ratio value $(\xi)$ is confirmed by Chen in [8]. In Figure $4, i_{F d}$ and $i_{F q}$ are actual compensation current compensated by SHAPF; $i_{F d}{ }^{*}$ and $i_{F q}{ }^{*}$ are reference currents calculated from Figure 3.

\section{Simulation Research}

In order to verify the effectiveness of the generalized inverse control policy, we use the MATLAB simulation platform to build a simulation study. And we do the comparison with feedforward PI control and inverse control. System parameters for simulation are shown in Table 1.

The SHAPF model is built as Figure 1 and the controller is applied as shown in Figure 3. The load current waveform is shown in Figure 5.
TABLE 1: SHAPF and controller parameter.

\begin{tabular}{lc}
\hline System parameters & value \\
\hline $\begin{array}{l}\text { Virtual value of grid voltage }\left(V_{m}\right) \text { and } \\
\text { frequency }\end{array}$ & $220 \mathrm{~V} / 50 \mathrm{~Hz}$ \\
$\begin{array}{l}\text { Equivalent inductance on grid }\left(L_{s}\right) \\
\text { Passive filter parameters } C_{f}, L_{f}, R_{f}\end{array}$ & $0.01 \mathrm{mH}$ \\
$\begin{array}{l}\text { DC side capacitor }\left(C_{\mathrm{dc}}\right) \text { and its voltage } \\
\left(V_{\mathrm{dc}}\right)\end{array}$ & $0.01 \mathrm{~F} / 480 \mathrm{~V}$ \\
PWM frequency & $10000 \mathrm{~Hz}$ \\
PI parameter of generalized inverse & $10000 / 2000,10000 / 2000$ \\
system $\left(k_{p 1}, k_{i 1}, k_{p 2}, k_{i 2}\right)$ & \\
$\begin{array}{l}\text { Generalized inverse parameter } \\
\left(a_{11}, a_{10}, a_{21}, a_{20}\right)\end{array}$ & $0.01,1.41,0.01,1.41$ \\
PI controller parameter in DC side \\
$\left(k_{p}, k_{i}\right)$
\end{tabular}




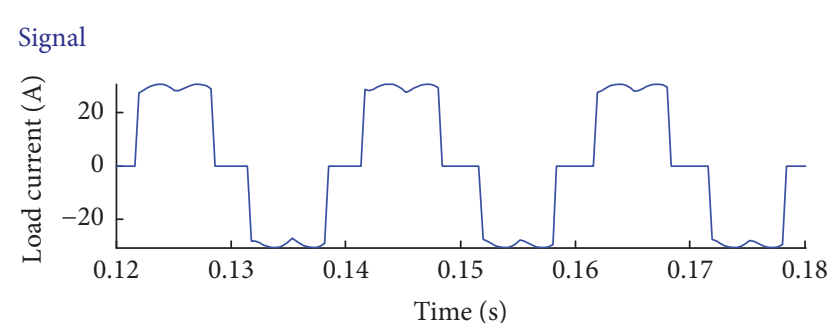

(a)

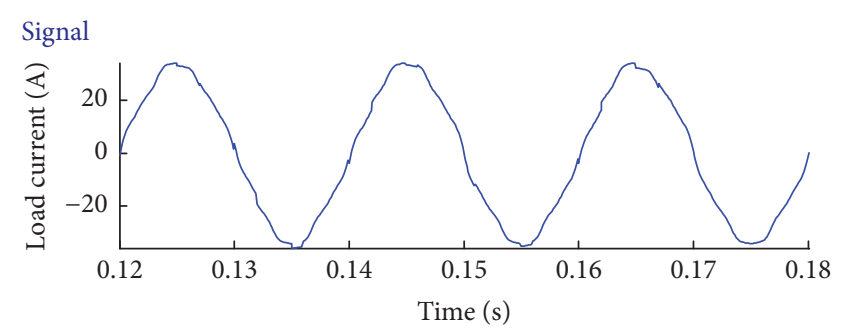

(c)

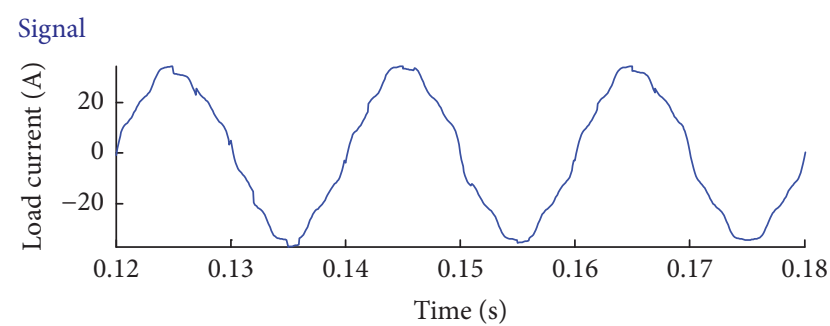

(b)

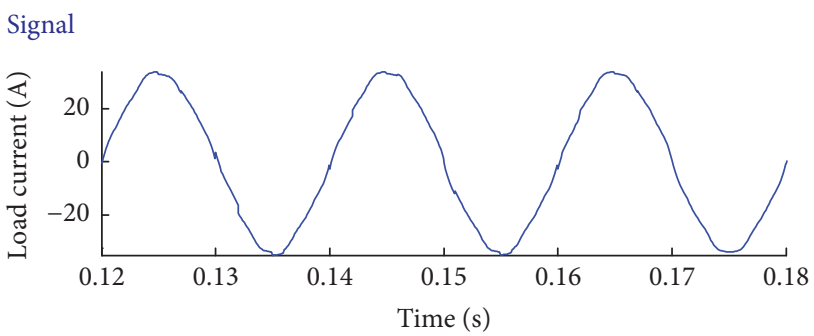

(d)

FIGURE 5: Load current waveform comparison. (a) Load current waveform without decoupling method. (b) Load current waveform with feedforward decoupling method. (c) Load current waveform with inverse system decoupling method. (d) Load current waveform with generalized inverse system decoupling method.

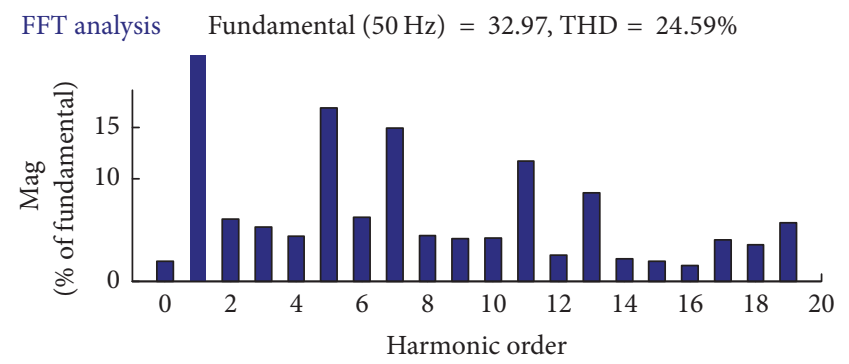

(a)

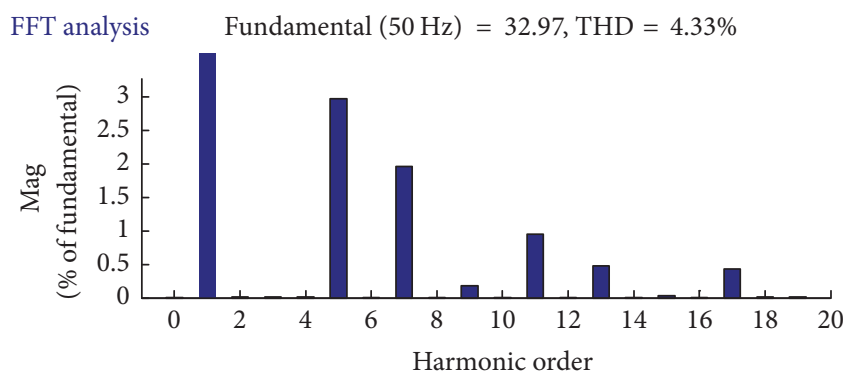

(c)

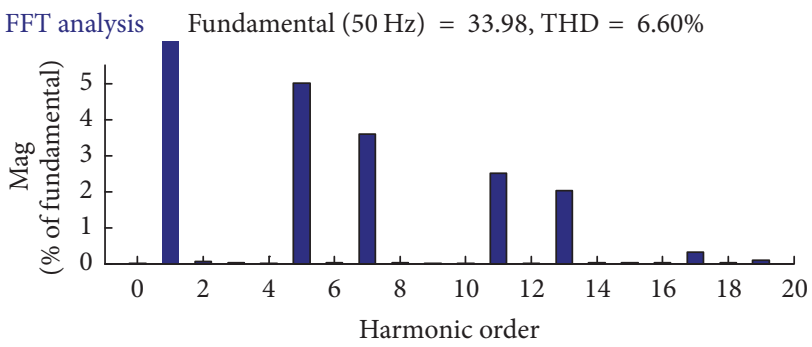

(b)

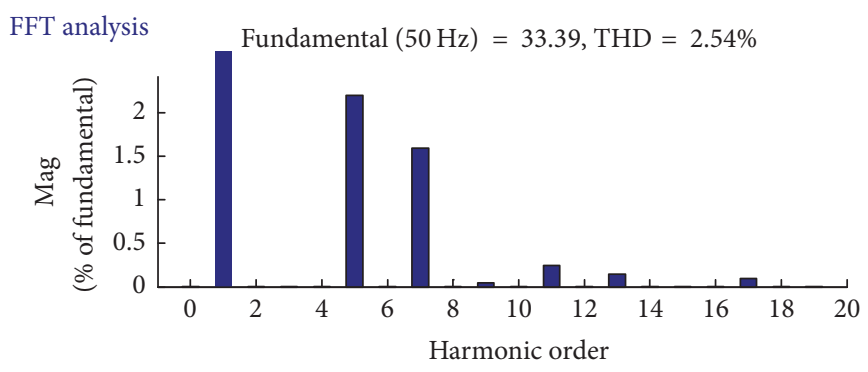

(d)

FIGURE 6: Spectrum analysis comparison. (a) Spectrum analysis of load current without decoupling method. (b) Spectrum analysis of load current with feedforward decoupling method. (c) Spectrum analysis of load current with inverse system decoupling method. (d) Spectrum analysis of load current with generalized inverse system decoupling method.

It is obvious that the load compensated waveform with generalized inverse system method is most close to ideal sine wave. The spectrum analysis is shown in Figure 6.

In Figure 6, the THD (total harmonic distortion) of the system without compensation device is $24.59 \%$. The THD of the system with traditional feedforward PI controller is $6.60 \%$. The THD of the system with inverse PI controller is $4.33 \%$. The THD of the system with generalized inverse PI controller is $2.54 \%$; each controller can compensate the harmonics on grid, but the generalized inverse controller has 


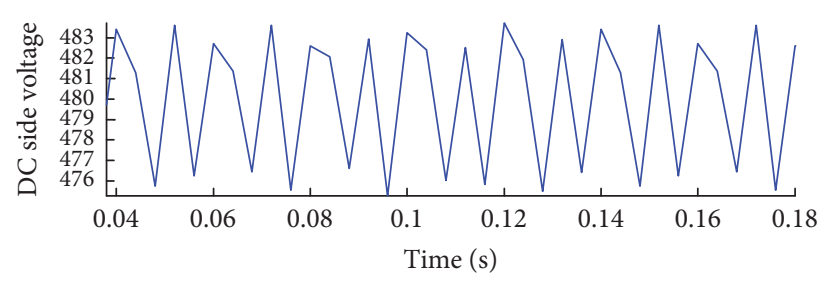

FIGURE 7: Voltage of DC side capacitor $\left(V_{\mathrm{dc}}\right)$.

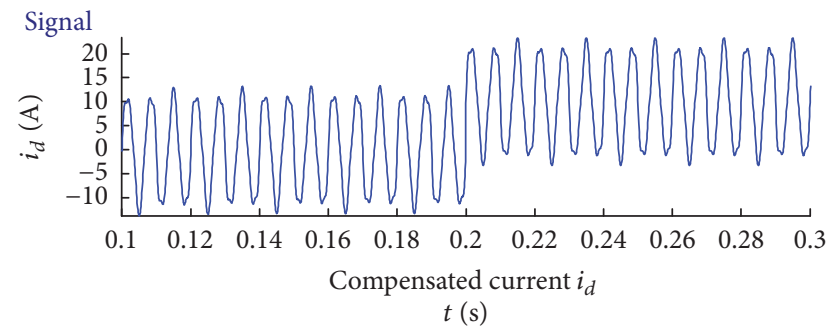

(a)

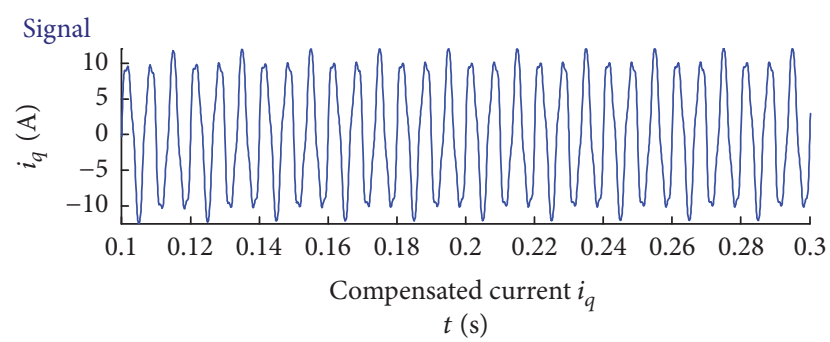

(b)

FIGURE 8: Compensated current in $d$-q coordinate. It is proved that $i_{d}$ and $i_{q}$ are completely decoupled in $d$ - $q$ coordinates.

the best effect: it can suppress the 5-order harmonic less than $2 \%$ of fundamental. Voltage of DC side capacitor is shown in Figure 7.

In Figure 7, the fluctuation of voltage of DC side capacitor is less than $4 \mathrm{~V}$ with PI controller. In order to prove that the system is completely decoupled, disturbance step signal is applied to $i_{d}$ at time of 0.2 seconds of the simulation. Waveform of $i_{d}$ and $i_{q}$ is shown in Figure 8.

It is easy to see that $i_{q}$ is not affected by the disturbance step signal on $i_{d}$. So the complex system is completely decoupled. The generalized inverse system decoupling method for SHAPF is effective.

\section{Conclusion}

In this paper, a novel method is presented to achieve the decoupling control of SHAPF; generalized inverse theory is applied to the system so that we can configure the pole parameters to adjust the dynamic performance of the system. The generalized inverse system is connected in series with SHAPF and the pseudolinear system can be decoupled into two linear systems to achieve the decoupling control. Decoupled subsystems can be easily bridled by PI controller. The simulation research proves that the system is completely decoupled and the compensation effect is better than the traditional PI control system and inverse system. This method can be used in reality for further research.

\section{Conflicts of Interest}

The authors declare that they have no conflicts of interest.

\section{Acknowledgments}

This work was supported by the National Natural Science Foundation of China under Project 61165006, the Science
Foundation of Gansu Province of China under Project 145RJZA182, the Large Electric Drive System and State Key Laboratory of Equipment Technology Openness Foundation of China under Project SKLLDJ022016015, and a Project Funded by the Colleges Fundamental Research under Project 214144 .

\section{References}

[1] Z. A. Wang and J. J. Liu, Harmonic Suppression and Reactive Power Compensation, China Machine Press, 2015.

[2] A. Abouloifa, F. Giri, I. Lachkar, F. Z. Chaoui, M. Kissaoui, and Y. Abouelmahjoub, "Cascade nonlinear control of shunt active power filters with average performance analysis," Control Engineering Practice, vol. 26, pp. 211-221, 2014.

[3] Z. B. Zou and L. J. Cai, "Active power filter nonlinear decoupling control," Automation of Electric Power Systems, vol. 14, no. 1, pp. 37-41, 2004.

[4] W. Luo and Y. Zhang, "Power Filter Based on current decouple control," Light Technology, vol. 8, no. 1, pp. 42-46, 2014.

[5] M. Benchouia, I. Ghadbane, A. Golea, K. Srairi, and M. Benbouzid, "Implementation of adaptive fuzzy logic and PI controllers to regulate the DC bus voltage of shunt active power filter," Applied Soft Computing, vol. 28, no. 1, pp. 125-131, 2015.

[6] X. Z. Deng, Neural Network Inverse Control Method for Multivariable Nonlinear Systems, Science Press, 2004.

[7] W. Lu, C. W. Li, and C. B. Xu, "Inverse system method based decoupling control of shunt hybrid active power filter," Control Theory \& Applications, vol. 9, no. 1, pp. 1145-1152, 2013.

[8] B. S. Chen, Electric Power Drag Automation Control SystemMotion Control System, China Machine Press, 2003. 


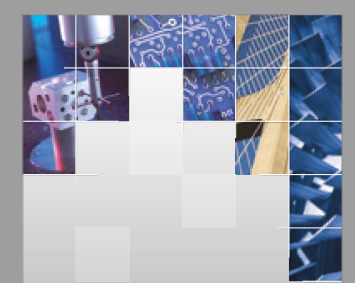

\section{Enfincering}
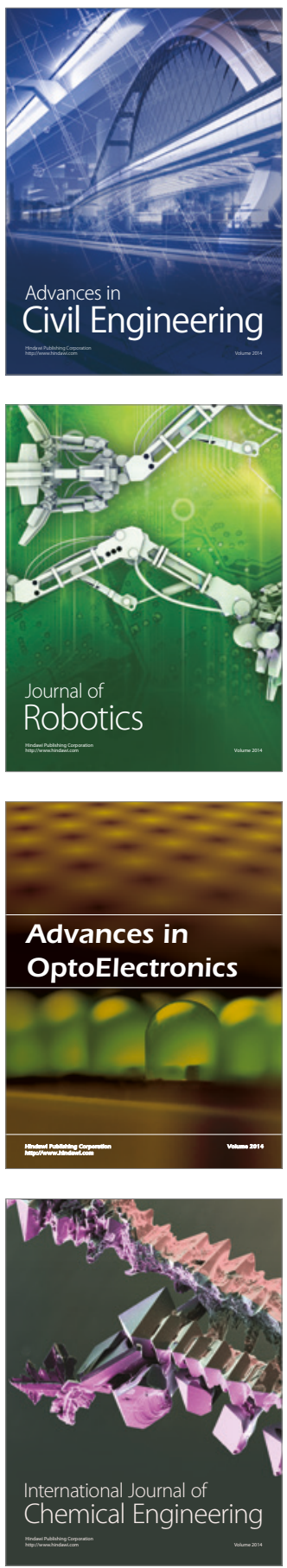

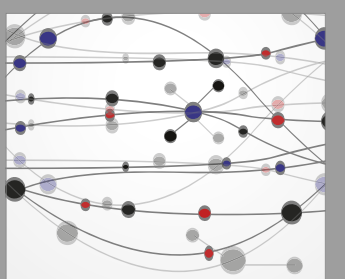

The Scientific World Journal

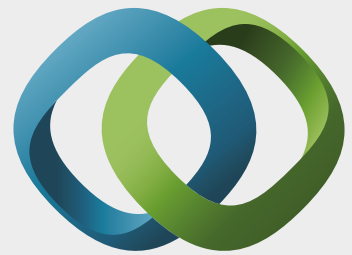

\section{Hindawi}

Submit your manuscripts at

https://www.hindawi.com
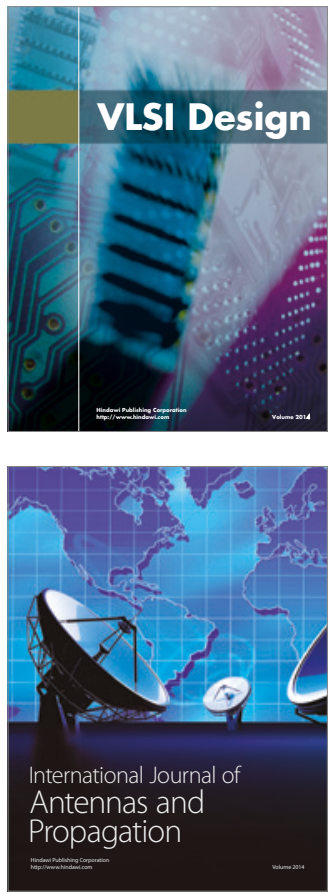

\section{Rotating}

Machinery
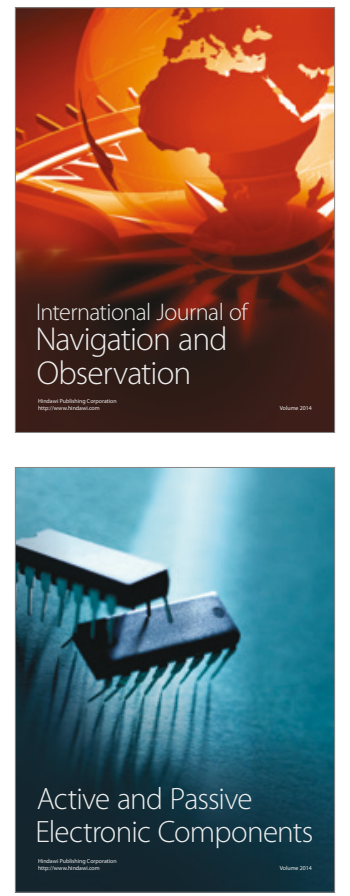
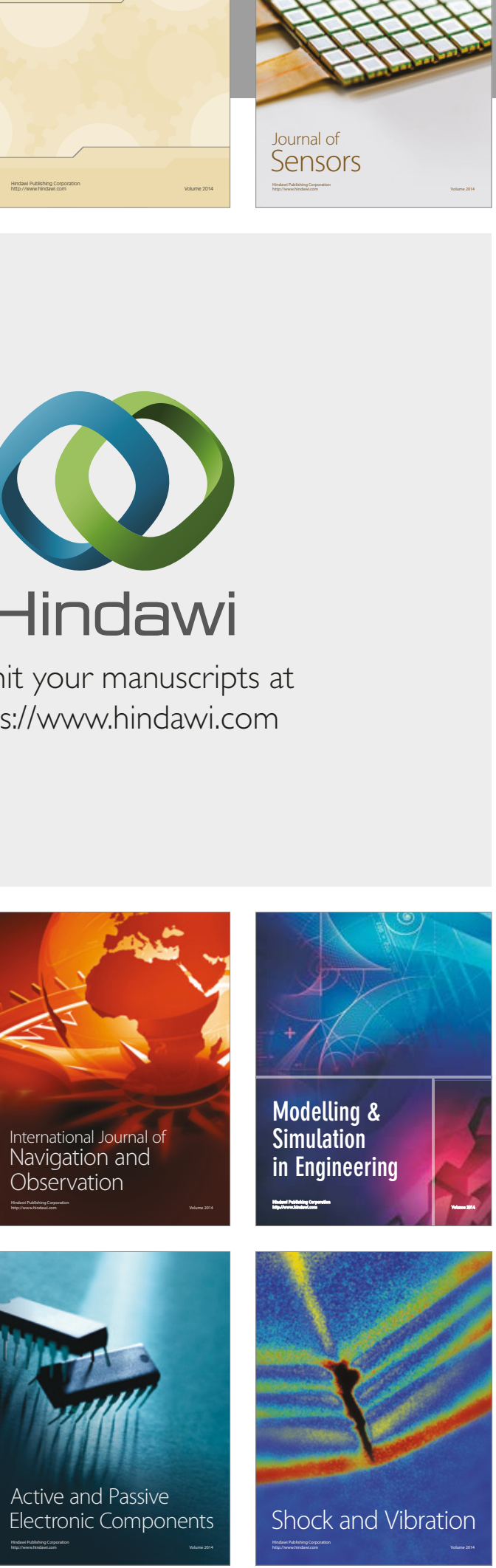
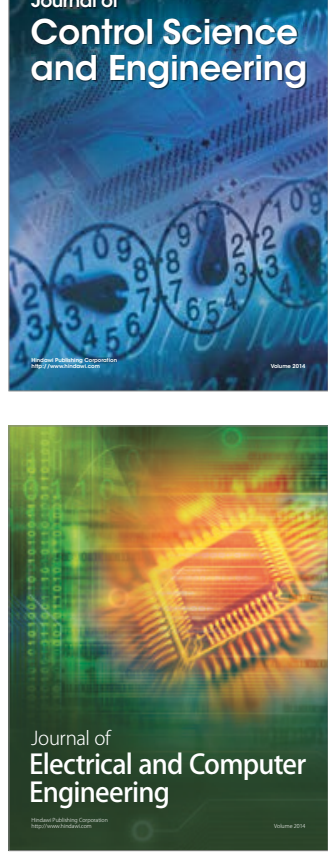

Distributed

Journal of

Control Science

and Engineering
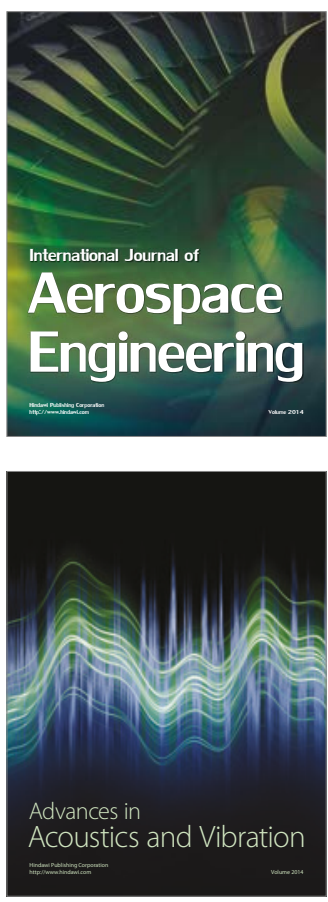

Sensor Networks 